\title{
Masses detection and classification in ultrasound images
}

\author{
${ }^{1}$ K.Subashini, ${ }^{2}$ K.Jeyanthi \\ ${ }^{I}$ PG Student, Dept. of ECE, K.P.R Institute of Engineering \& Technology, India \\ ${ }^{2}$ Assistant Professor, Dept. of ECE, K.P.R Institute of Engineering \& Technology, India
}

\begin{abstract}
Breast cancer is the second leading cause of death for women all over the world. Since the cause of the disease remains unknown, early detection and diagnosis is the key for breast cancer control, and it can increase the success of treatment and save lives. Ultrasound imaging is one of the most frequently used diagnosis tools to detect and classify abnormalities of the breast. In order to eliminate the operator dependency and improve the diagnostic accuracy, computer-aided diagnosis $(C A D)$ system is a valuable and beneficial means for breast cancer detection and classification. The performance of the CAD system is fairly evaluated. Generally, a CAD system consists of four stages: preprocessing, image segmentation, feature extraction and selection, and classification. Masses and microcalcification are both important signs of breast cancer. In this paper, masses are identified and classified into benign and malignant.
\end{abstract}

Keywords: computer aided diagnosis (CAD), breast ultrasound (BUS), feature extraction and selection, classifiers.

\section{Introduction}

Breast cancer is the second leading cause of death for women all over the world and more than $8 \%$ women will suffer this disease during their lifetime. In 2008, there were reported approximately 182,460 newly diagnosed cases and 40,480 deaths in the United States. Since the causes of breast cancer still remain unknown, early detection is the key to reduce the death rate. The earlier the cancers are detected, the better treatment can be provided. A good detection approach should produce both low false positive (FP) rate and false negative (FN) rate. Previously, the most effective modality for detecting and diagnosing breast cancer is mammography, but limitations are there in mammography for breast cancer detection. Mammography can hardly detect breast cancer in adolescent women with dense breasts. In addition, the ionizing radiation of mammography can increase the health risk for the patients and radiologists.

Currently, an important alternative to mammography is ultrasound (US) imaging, and it shows an increasing interest in the use of ultrasound images for breast cancer detection. Studies have demonstrated that using US images can discriminate benign and malignant masses with a high accuracy. Use of ultrasound can increase overall cancer detection by $17 \%$ and reduce the number of unnecessary biopsies by $40 \%$. Breast ultrasound (BUS) imaging is superior to the mammography in the facts: (1) ultrasound is radiation free, more convenient and safer than mammography for patients and radiologists. (2) Ultrasound is more sensitive in detecting abnormalities. (3) There is a high rate of false positives in mammography which causes a lot of unnecessary biopsies. In contrast, the accuracy rate of BUS imaging in the diagnosis of simple cysts can reach 96-100\%. Most of the CAD systems need a large number of samples to construct the models or rules, but proposed a novel diagnosis system requiring very few samples. Generally, the ultrasound CAD systems for breast cancer detection involve four stages as shown in Fig.1.

\section{A. Image preprocessing}

The major limitations of BUS imaging are the low contrast and interference with speckle. The task of image preprocessing is to enhance the image and to reduce speckle without destroying the important features of BUS images for diagnosis.

\section{B. Image segmentation}

Image segmentation divides the image into non-overlapping regions, and it will separate the objects from the background.

\section{Feature extraction and selection}

This step is to find a feature set of breast cancer lesions that can accurately distinguish lesion/non lesion benign or malignant and plays an important role in feature extraction for diagnosis. The feature space could be very large and complex, so extracting and selecting the most effective features is very important. 


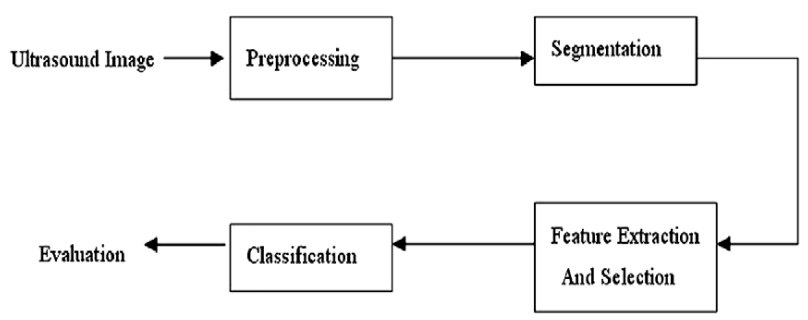

Fig.1. CAD system for breast cancer

\section{Classification}

Based on the selected features, he suspicious regions will be classified as benign or malignant by the classification methods.

The advantage of CAD system is its simple structure and fast processing speed, and disadvantage is that the features extracted directly from ROIs may not provide robust and accurate performance.

\section{Preprocessing}

The preprocessing of BUS images consists of speckle reduction and image enhancement. Speckle is a form of multiplicative noise generated by a number of scatters with random phase within the resolution cell of ultrasound beam has demonstrated that the $k$-distribution is a good model for the amplitude distribution of the received signal. A more generalized statistical model, the homodyned $k$-distribution, has been analyzed. It combined the features of the $k$-distribution and Rice distribution to better account for the statistics of the signal. To detect speckles, the parameters for the speckles should be estimated first. The speckle parameters of the $k$ distribution model can be estimated based on the moments. An iterative method using the statistics of ultrasound signal is proposed to find the parameters of the homodyned $k$-distribution model. Speckle makes the visual observation and interpretation difficult. Therefore, removing speckle without destroying important features for diagnosis is critical. Some speckle reduction techniques only work well on additive noise, and logarithmic compression is often employed to convert multiplicative noise into additive noise. Image enhancement is used to improve the quality of low contrast images. We will review speckle reduction and image enhancement separately, however, many techniques can achieve both goals at the same time.

\section{Wavelet domain techniques}

The discrete wavelet transform (DWT) translates the image into an approximation sub-band consisting of the scale coefficients and a set of detail sub-bands at different orientations and resolution scales composed of the wavelet coefficients. DWT provides an appropriate basis for separating the noise from an image. As the wavelet transform is good at energy compaction, the small coefficients more likely represent noise, and large coefficients represent important image features. These properties make DWT attractive for denoising. The general procedure is: (1) calculate the discrete wavelet transform; (2) remove noise by changing the wavelet coefficients; and (3) apply the inverse wavelet transform (IDWT) to construct the despeckled image. The techniques are grouped as: (1) wavelet shrinkage; (2) wavelet despeckling under Bayesian framework; and (3) wavelet filtering and diffusion. Wavelet shrinkage method is chosen for wavelet domain technique.

\section{Wavelet shrinkage}

The wavelet shrinkage is based on thresholding the wavelet coefficients. It suppresses the coefficients representing noise while retains the coefficients that more likely representing image features. It is usually performed using one of the two dominant thresholding schemes: hard thresholding and soft thresholding. Suppose the image in wavelet domain is represented as

$$
o=s+n
$$

Where $o$ is the observed wavelet coefficients, $s$ is the noise free component and $n$ is the additive noise. The wavelet shrinkage estimator can be represented as

$$
s=H o
$$

where $H$ denotes shrinkage factor. For the classical wavelet thresholding rules, a threshold value $T$ is defined and $H$ is specified as follows.

For hard thresholding

For soft thresholding

$$
\begin{array}{r}
H=\{0 \text { if }|o|<T \\
1 \text { if }|o| \geq T
\end{array}
$$

$$
\begin{aligned}
& H=\{0 \text { if }|o|<T \\
& 1-T / o \mid \text { if }|o| \geq T
\end{aligned}
$$


Where $|o|$ denotes the absolute value of $o$. Adaptive thresholding which makes threshold values adaptive to the spatially changing statistics of the images has attracted more attention. Adaptive thresholding improves the performance by incorporating additional local information such as the identification of edge into the despeckling algorithm.

\section{Segmentation}

Segmentation is a partition of the image into non- overlapping regions. The goal for the segmentation is to locate the suspicious areas to assist radiologists in diagnoses.

\section{Active contour model}

The active contour model, more widely known as snake, is a framework for delineating an object outline from a possibly noisy 2D image, and has been massively used as an edge based segmentation method. This approach attempts to minimize the energy associated with the current contour as the sum of the internal and external energies. The snake model modifies its shape actively and approximates the desired contour. During the deformation process, the force is calculated from the internal energy and external energy. The external energy derived from image feature energy is used to extract the contour of the desired object boundary. The internal energy derived from the contour model is used to control the shape and regularity of the contour. The snake model has been extensively used for US images.

Combining intensity and texture with empirical domain-specific knowledge and directional gradient, a deformable shape based model was studied to find lesion margins automatically. A formulation of the empirical rules used by radiologists in detecting ultrasonic breast lesions was employed to automatically determine a seed point in the image. Followed by region growing to obtain an initial segmentation of the lesion, image pixels were classified according to the intensity and texture. Finally, the image was segmented according to the minimum energy.

\section{Feature Extraction and Selection}

Feature extraction and selection are important steps in breast cancer detection and classification. An optimum feature set should have effective and discriminating features, while mostly reduce the redundancy of feature space to avoid curse of dimensionality. The features of breast US images can be divided into four categories: texture, morphologic, model-based and descriptor features. Extraction and selection of effective features is a necessary step. The features extraction and selection mainly include four considerations: discrimination, reliability, independence and optimality. The goal of feature extraction and selection is to maximize the discriminating performance of the feature group.

\section{Texture features}

Most of the texture features are calculated from the entire image or ROIs using the gray level values. Auto-covariance coefficient is a basic and traditional texture feature which can reflect the inner pixel correlation with in an image. Distribution distortion of wavelet coefficients is defined as the summation of differences among the real distribution of wavelet coefficients in each high frequency sub-band and distribution of the expected Laplacian distribution. The contrast, energy, entropy and inverse difference normalized values are calculated from the formulae

Energy $(E)=p(x, y)^{\wedge} 2$

It is a gray-scale image texture measure of homogeneity Changing, reflecting the distribution of image gray-scale uniformity of weight and texture

Contrast $(\mathrm{I})=\Sigma \Sigma(\mathrm{x}-\mathrm{y}) 2 \mathrm{p}(\mathrm{x}, \mathrm{sy})$

Contrast is the main diagonal near the moment of inertia, which measure the value of the matrix is distributed and images of local changes in number, reflecting the image clarity and texture of shadow depth. Contrast is large means texture is deeper.

$$
\text { Entropy }(\mathrm{S})=\Sigma \Sigma \mathrm{p}(\mathrm{x}, \mathrm{y}) \log \mathrm{p}(\mathrm{x}, \mathrm{y})
$$

Entropy measures image texture randomness, when the space co-occurrence matrix for all values are equal, it achieved the minimum value, if the value of co-occurrence matrix is very uneven, its value is greater. Therefore, the maximum entropy implied by the image gray distribution is random.

Inversedifference $(\mathrm{H})=\Sigma \Sigma\left(1 /\left(1+(\mathrm{x} y)^{\wedge} 2\right)^{*} \mathrm{P}(\mathrm{x}, \mathrm{y})\right.$

It measures local changes in image texture number. Its value in large is illustrated that image texture between the different regions of the lack of change and partial very evenly. Here $p(x, y)$ is the gray-level value at the coordinate $(\mathrm{x}, \mathrm{y})$ 


\section{Classification}

After the features have been extracted and selected, they are input into a classifier to categorize the images into lesion/non-lesion or benign/malignant classes. Lesion detection is necessary before lesion classification. Artificial neural network is used to classify the images into benign or malignant because artificial neural network has no rule, widely applicable and robustness.

\section{Artificial neural network}

Artificial neural networks are the collection of mathematical models that imitate the properties of biological nervous system and the functions of adaptive biological learning. ANN is composed of an input layer, an output layer and one or more hidden layers. Back-propagation neural network is used in ANN to classify the images.

\section{Back-propagation neural network}

Back-propagation neural network is a feed-forward ANN with supervised learning process. It is widely applicable and no rule is needed. Frequently used back-propagation neural networks have one or two hidden layers and 2-10 neurons in each layer. There is no universal rule to decide the number of layers or number of neurons in each layer. The Back-Propagation neural network was used in the proposed CAD and the result was compared with the linear classifier. The training samples for Back-Propagation neural network and only those samples within a distance to the cluster center would be used for training. The performance of back-propagation neural network is better than that of linear classifiers.

\section{Evaluation}

The ultrasound CAD system is evaluated fairly and correctly, based on the classifiers chosen. Thus the masses are classified into benign and malignant. The performance of the CAD system is evaluated.

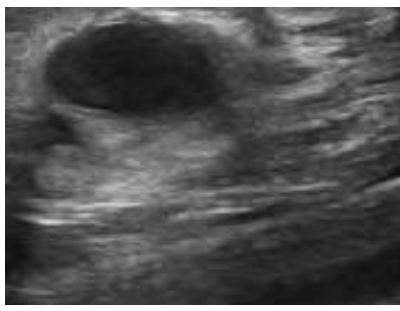

Fig.2. Benign mass

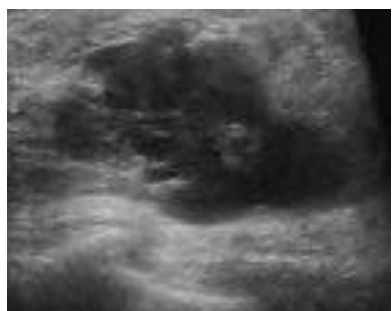

Fig.3. Malignant mass

\section{Conclusion}

Breast ultrasound is a cancer detection method which is non-invasive, non-radiating, passive, fast, painless, low cost, risk free. Breast ultrasound is suitable for women of all ages, including pregnant or nursing women, with all sizes of breast, with or without breast implants, fibrocystic breasts. Thus it overcome the disadvantages of mammography and other existing methods.

we detected the masses of the sample breast ultrasound images and classified into benign or malignant. In future microcalcification will be detected and classified into benign or malignant using ultrasound images.

\section{References}

[1]. A.shahzad, M. O’Halloran, M. Glavin, Prefiltered Beamforming for Early-Stage Breast Cancer Detection .vol 12,2013.

[2]. Li Xu, Xia Xiao, Takamaro Kikkawa, Ultra-wide band microwave image reconstruction for early breast cancer detection by norm constrained capon beamforming, mathematical and computer modelling 58(2013) 403-408.

[3]. Tanja Cufer, Fatima cardoso, Gustavo werutsky, Herve Bonnefoi, Etienne brain, David Cameron, The EORTC Breast cancer group: major achievements of 50 years of research and future directions.ejc supplements, no (2012) 27-33.

[4]. sudeshna gangopadhyay, Argha Nandy, Pooja Hor, Ashis Mukhopadhyay, Breast Cancer Stem Cells: A Novel Therapeutic Target, Clinical Breast Cancer,vol.13,No.1,7-15(2013)

[5]. Xiangjun shi, H.D.cheng, Liming Hu, Wen Ju,Jiawei Tian, Detection and Classification of masses in breast Ultrasound Images, Digital Signal processing, volume 20, Issue 3, may 2010, pages 824 -836.

[6]. Shafimirza, Dr.j.Apparao, Retrieval of digital images using texture feature with advanced genetic algorithm, volume31, issue42012.

[7]. Afsaneh Jalalian, Syamsiah B.T. Mashohor,Hajjah Rozi mahmud, M.Iqbal B. Saripan, Abdul rahman B.Ramli, Babak Karasfi. Computer-aided detection /diagnosis of breast cancer in mammography and ultrasound. Clinical imaging 37 (2013) $420-426$.

[8]. Paul Hamberg, Monique M.E.M. Bos, Hans J.J. Braun, Jacqueline M.L. Stouthard. Randomized Phase II study Comparing Efficiency and Safety of Combination. Oct 2010 